demographics, fracture etiology and characteristics, medical comorbidities, procedure characteristics, procedure-related complications, resource utilization outcomes, pain and disability outcomes, as well as quantitative measures of radiographic endpoints obtained from follow up spine CT studies. Restoration of vertebral height, as determined by the vertebral body height ratio, is the primary endpoint. Secondary enpoints include pain reduction measured by VAS score, functional improvement measured by the Owestry Disability Index, and the rate of adjacent level fractures. Complete datasets will be collected from cases pre-operatively, as well as 1, 3, and 6 months post-operatively.

Expected Outcomes We anticipate observing that the SpineJack achieves adequate restoration of vertebral height with shorter procedure times and smaller volumes of cement relative to previously reported values for other vertebral augmentation described in the literature. We also expect to observe significant and sustained improvement in both pain and function scores at pre-determined time points up to at least 6 months post-operation compared to baseline scores measured preoperatively.

Discussion With a strong mechanistic rationale based on the promising findings from a previous prospective study carried out in Europe, this study will provide among the first comprehensive characterizations of early experiences with the SpineJack in the United States. As with any early experiences with a novel technology, key insights that improve future use of the technology may be gleaned from rigorous analysis of thoroughly collected data.

Disclosures M. Martini: None. K. Yaeger: None. D. Nistal: None. A. Doshi: None. R. De Leacy: None.

\section{E-077 STEM CELL THERAPY FOR SPINAL CORD INJURY}

W Kamel Sr*. Immunology, Egyptian Military Medical Academy, Cairo, Egypt

10.1136/neurintsurg-2019-SNIS.152

Spinal cord injuries (SCI) cause sensory loss and motor paralysis and are treated with physical therapy, but most patients fail to recover due to limited neural regeneration. Here we describe a strategy in which treatment with autologous adherent bone marrow cells is combined with physical therapy to improve motor and sensory functions in earlystage chronic SCI patients. In a phase I/II controlled singleblind clinical trial (clinicaltrials.gov identifier: NCT00816803), 70 chronic cervical and thoracic SCI patients with injury durations of at least 6 months were treated with either intrathecal injection(s) of autologous adherent bone marrow cells combined with physical therapy, or with physical therapy alone. Patients were evaluated with clinical examinations, electrophysiological somatosensory evoked potential, MRI imaging, and functional independence measurements. Chronic cervical and thoracic SCI patients treated with autologous adherent bone marrow cells combined with physical therapy showed functional improvements over patients in the control group treated with physical therapy alone, and there were no cell therapy-related side effects. At 18 months posttreatment, 23 of the 50 cell therapy-treated cases $(46$ percent) showed sustained improvement using the American Spinal Injury Association (ASIA) Impairment Scale (AIS). Compared to those patients with cervical injuries, a higher rate of functional improvement was achieved in thoracic SCI patients with shorter durations of injury and smaller cord lesions. Therefore, when combined with physical therapy, autologous adherent bone marrow cell therapy appears to be a safe and promising therapy for patients with chronic spinal cord injuries. Randomized controlled multicenter trials are warranted.

Disclosures W. Kamel: None.

\section{E-078 NEURO IR INTRANET WEBSITE IS ASSOCIATED WITH IMPROVEMENT IN STAKEHOLDER JOB FACILITATION, SELF-EFFICACY, AND SATISFACTION}

${ }^{1} Z$ Voronovich*, ${ }^{1} A$ Montes, ${ }^{2} D$ Sorte. ${ }^{1}$ Department of Neurosurgery, University of New Mexico, Albuquerque, NM; ${ }^{2}$ Department of Neurosurgery and Radiology, University of New Mexico, Albuquerque, NM

\subsection{6/neurintsurg-2019-SNIS.153}

Introduction Neurointerventional radiology (NIR) provides a challenging environment for communication and performance as stakeholders come together from different teams to successfully execute NIR cases. Although success in NIR requires a high level of domain-specific expertise from the team members, there are no nurses, radiology technologists, or operating room technologists dedicated to NIR at our institution, and this arrangement is likely encountered at other hospitals. Such challenges can lead to poor confidence and low satisfaction on the job. In an effort to improve communication and support the performance of our NIR stakeholders, we developed an intranet website based on the Microsoft SharePoint platform. The website provides preference cards, links to relevant articles, device information, policies, case-specific data collection forms, and tracking for areas of improvement. The software was available to us through institutional licensing without additional cost. The website was created by a neurosurgical resident and the NIR clinical coordinator over a period of a month, and is primarily maintained by the clinical coordinator. We assessed the impact of the website on stakeholder job facilitation, self-efficacy, and satisfaction using surveys administered prior to and after the deployment of the site.

Methods We distributed a series of three anonymous internetbased surveys to 60 identified NIR stakeholders immediately prior to the deployment of the website, one month after the deployment, and four months after the deployment. We built our survey based on previously validated survey instruments from the information systems adoption literature. We included two questions on job self-efficacy, three questions on job facilitation, and three questions on job satisfaction, all of which were graded on a five-point Likert scale. Awareness of the website, frequency of website use, and recent NIR case participation were also assessed using self-reported scales. Questions addressing gender, age, and NIR role were optional due to concerns for preserving anonymity of the respondents.

Results The response rates were 52\%, 34\% and 35\% for the pre-deployment, one-month, and four-month surveys, respectively. $77 \%$ of active NIR case participants reported recently using the website one month after deployment, and the reported rate of active use increased to $85 \%$ at four months. There was a statistically significant improvement in self-efficacy one month after website deployment $(p=0.05)$, while self-rated possession of knowledge and task independence 
increased by $11 \%$ and $17 \%$, respectively, after four months. Job facilitating conditions and job satisfaction also demonstrated statistically significant improvement at four months $(\mathrm{p}=0.01$ and $\mathrm{p}=0.04$, respectively).

Concluion An intranet website for knowledge sharing can be an easily-deployed and inexpensive way to support improvement in job facilitation, self-efficacy, and job satisfaction in NIR. Such a website is likely to be beneficial in other dynamic procedural environments without dedicated staff. The study was limited by lack of controls for other work environment changes, such as staff turn-over, anonymous data collection preventing cross-linking of individual respondents across time, and utilization of self-reported adoption rates.

Disclosures Z. Voronovich: None. A. Montes: None. D. Sorte: None.

\section{E-079 TIME OF DAY AND ENDOVASCULAR TREATMENT DECISION IN ACUTE STROKE - INSIGHTS FROM AN INTERNATIONAL MULTIDISCIPLINARY SURVEY}

${ }^{1} \mathrm{~N}$ Kashani ${ }^{*},{ }^{2} \mathrm{~J}$ Ospel, ${ }^{1} \mathrm{~A}$ Wilson, ${ }^{3} \mathrm{~W}$ Kunz, ${ }^{4} \mathrm{P}$ Sylaia, ${ }^{5} \mathrm{~B}$ Baxter, ${ }^{6} \mathrm{~B}$ Campbell, ${ }^{7} \mathrm{U}$ Fischer, ${ }^{8} \mathrm{~A}$ Rabinstein, ${ }^{9} \mathrm{~S}$ Yoshimura, ${ }^{10} \mathrm{~J}$ Heo, ${ }^{11} \mathrm{~B}$ Kim, ${ }^{12} \mathrm{M}$ Cherian, ${ }^{13} \mathrm{~F}$ Turjman, ${ }^{1} \mathrm{M}$ Foss, ${ }^{1} \mathrm{~B}$ Menon, ${ }^{14} \mathrm{G}$ Saposnik, ${ }^{1} \mathrm{M}$ Goyal. ${ }^{1}$ Radiology, University of Calgary, Calgary, $A B$, Canada; ${ }^{2}$ Radiology, University Hospital Basel, Basel, Switzerland; ${ }^{3}$ Radiology, University Hospital Munich, Munich, GERMANY; ${ }^{4}$ Radiology, University of Calgary, Thiruvananthapuram, India; ${ }^{5}$ Radiology, Erlanger Hospital Chattanooga, Chattanooga, TN; ${ }^{6}$ Radiology, Royal Melbourne Hospital, University of Melbourne, Melbourne, Australia; ${ }^{7}$ Radiology, University Hospital Bern (Inselspital), Bern, Switzerland; ${ }^{8}$ Radiology, Mayo Clinic Rochester, Calgary, MN; ${ }^{9}$ Radiology, University of Calgary, Hyogo, Japan; ${ }^{10}$ Neurology, Yonsei University, Seoul, Korea, republic of; ${ }^{11}$ Radiology, St. Mary's Hospital Seoul, Calgary, Korea, republic of; ${ }^{12}$ Radiology, University of Calgary, Tamil Nadu, India; ${ }^{13}$ Radiology, Centre Hospitalier Universitaire de Lyon, Lyon, France; ${ }^{14}$ Radiology, University of Toronto, Calgary, ON, Canada

\subsection{6/neurintsurg-2019-SNIS.154}

Background The decision to proceed with endovascular thrombectomy should ideally be made independent of inconvenience factors such as daytime, especially in cases supported by level IA evidence. The treatment decision may be influenced by the time of day when facing level 2B evidence cases. We assessed the influence of patient presentation time on EVT decision- making under current local resources and assumed ideal conditions.

Methods In an international cross-sectional survey among 607 stroke physicians, participants were asked to give their treatment decisions to 10 out of 22 randomly assigned case scenarios, 8 of them were level $1 \mathrm{~A}$ evidence cases (all daytime scenarios), 11 were scenarios where level $2 \mathrm{~B}$ evidence supports EVT, among them 7 daytime scenarios (8:00 am5:00 pm) and 4 overnight cases (10 pm:00 - 7:00 am). The remaining 3 ( 2 daytime and 1 nighttime) cases were not covered by current treatment guidelines. Participants stated A) their treatment approach assuming there were no practice constraints, and B) the treatment they would pursue under their current local resources. Endovascular treatment decision in level $2 \mathrm{~B}$ scenarios was analyzed with regard to presentation time.

Results 3034 responses for 12 case scenarios from 607 physicians from 38 countries with level $2 \mathrm{~B}$ evidence and 2208 for level 1A evidence for EVT were received. In the subgroup of level 2B evidence scenarios, participants selected EVT more often in the overnight cases (in $74.2 \%$ under assumed ideal conditions and in $70.7 \%$ under current local resources) than in the daytime scenarios (in $67.2 \%$ and $63.8 \%$ respectively). Overnight presentation did not increase the risk for a treatment decision against EVT. Current nighttime EVT decision rates for level $2 \mathrm{~B}$ scenarios in 7 countries were $>10 \%$ lower than ideal nighttime EVT decision rates.

Conclusion Overall, overnight presentation did not increase the risk of a treatment decision against EVT when facing level 2B evidence. Hence, presentation time is unlikely to influence endovascular treatment decision-making in this subset of stroke patients. Current nighttime EVT decision rates in 7 countries were substantially lower than ideal nighttime EVT rates, potentially indicating limited access to endovascular treatment beyond working hours.

Disclosures N. Kashani: None. J. Ospel: None. A. Wilson: None. W. Kunz: None. P. Sylaia: None. B. Baxter: None. B. Campbell: None. U. Fischer: None. A. Rabinstein: None. S. Yoshimura: None. J. Heo: None. B. Kim: None. M. Cherian: None. F. Turjman: None. M. Foss: None. B. Menon:None. G. Saposnik: None. M. Goyal: None.
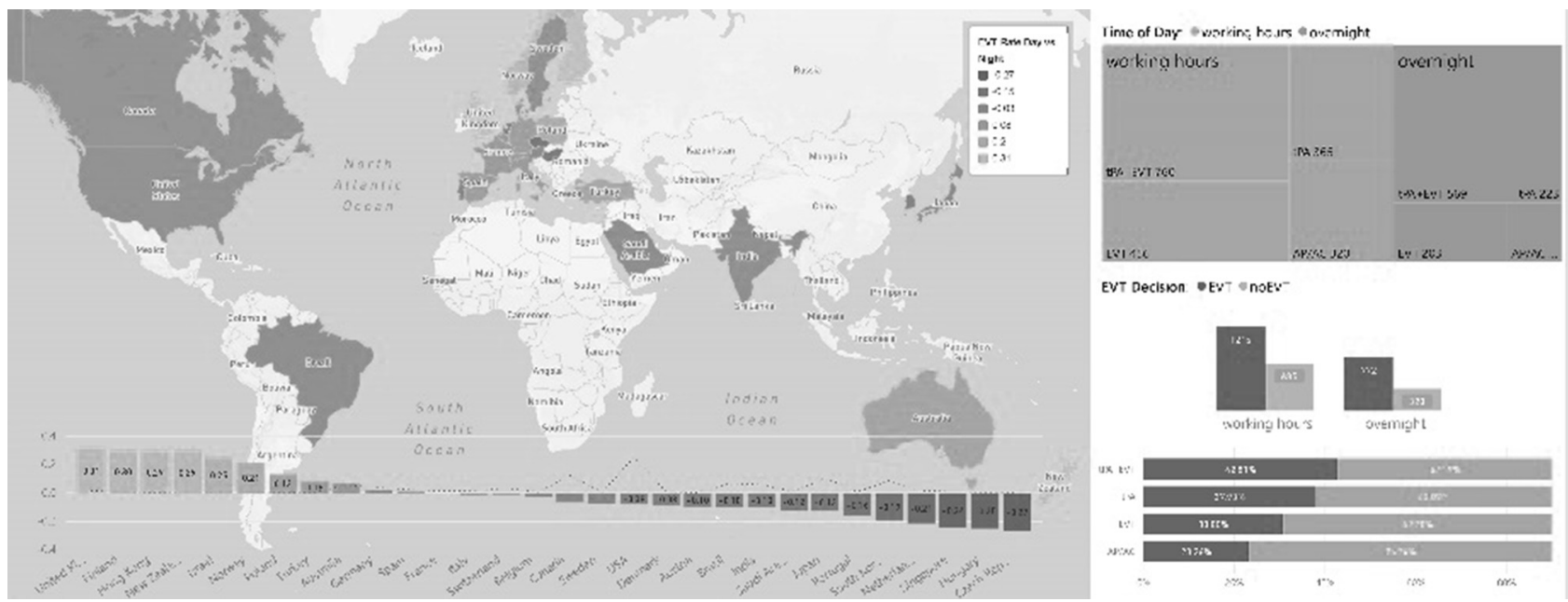

Abstract E-079 Figure 1 